\title{
An Excitation Signal Source with Capability of Temperature-drift Suppression for Eddy Current Detection
}

\author{
Jiang Guodong \\ School of Energy and Electrical Engineering \\ Nanjing Institute of Industry Technology, Nanjing, 210046, \\ China
}

\begin{abstract}
An excitation signal source which is capable of suppressing temperature drifting is designed in this paper. The influence of temperature drifting of the amplifier on the stability and accuracy of data measurements is also studied. The mathematical model on which the design is based is introduced and discussed. The experimental results show that the design is effective in suppressing temperature drifting of the amplifier, therefore it significantly improves the consistency and accuracy of results when it is applied to the application of thickness measurement of PCB copper foil .
\end{abstract}

Keywords-Temperature-drifting, Integrating circuit, Lock-in amplifier, Excitation signal

\section{INTRODUCTION}

The eddy current detection is based on the theory of electromagnetic induction. When a changing magnetic field created by alternating current is applied to a test object, then the eddy current generates in the test object. The detection of surface cracks and the measurement of coating thickness on a test object can be accomplished by measuring the variations of the amplitude and phase of the eddy current. The alternating current is created by the excitation signal through modulation. The excitation signal is not only the carrier signal but the reference signal as well, and how stable and accurate it is would determine how accurate the measurement would be for eddy current testing. The excitation signal is mainly from a circuit consisting of op-amps. The theoretical analysis and experiments show that the output drifting in op-amps are mainly due to temperature drifting. The direct coupling is usually used between stages in op-amps, therefore the impact of temperature drifting would increase with the increases in the number of stages and the amplification factor, therefore it is very critical to create a negative feedback system with lowtemperature-drifting integrated circuits so that the excitation signal is consistent and accurate within certain range of temperature.

\author{
Chen Liyuan \\ School of Energy and Electrical Engineering \\ Nanjing Institute of Industry Technology, Nanjing, 210046, \\ China
}

\section{THE MATHEMATICAL MODEL OF A NEGATIVE FEEDBACK} SYSTEM T

Figure 1 shows the configuration of a negative feedback system. It uses the detecting module and integrating module. The deep-degree negative feedback is realized by using high DC gain in integrating circuit. The fundamental analysis of its working is shown in the following.

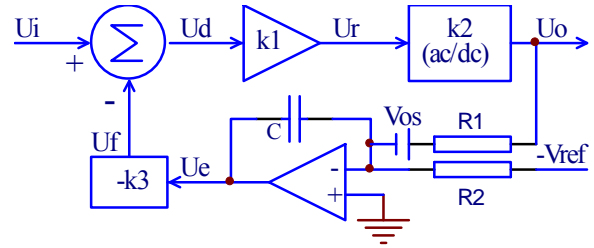

Figure.1 The mathematical model of the negative feedback system

The output is:

$$
u_{o}=k_{1} k_{2} u_{d}
$$

The integrating error is:

$$
u_{e}=-\left(\frac{u_{o}}{R_{1}}-\frac{V_{r e f}}{R_{2}}-\frac{V_{o s}}{R_{12}}\right) \times \frac{1}{\omega c}
$$

Where $V_{\text {os }}$ is the input offset, $R_{12}=R_{1} / / R_{2}$.

The Feedback signal is:

$$
u_{f}=-k_{3} u_{e}
$$

Difference signal:

$$
u_{d}=u_{i}-u_{f}
$$

Substituting (2), (3), and (4) into (1), one gets the (5):

$$
\begin{aligned}
& u_{o}=\left(k_{1} k_{2} u_{i}+k_{1} k_{2} k_{3} \times \frac{V_{r e f}}{R_{2} \omega C}+\right. \\
& \left.k_{1} k_{2} k_{3} \times \frac{V_{o s}}{R_{12} \omega C}\right) /\left(1+k_{1} k_{2} k_{3} \times \frac{1}{R_{1} \omega C}\right)
\end{aligned}
$$

Since $u_{o}$ is a DC signal, when $\omega \rightarrow 0, \frac{1}{\omega C} \rightarrow \infty$, then: 


$$
u_{o}=\frac{V_{r e f}}{R_{2}}+\frac{V_{o s}}{R_{12}}
$$

From (6) one can tell that $u_{o}$ is only associated with $R_{1}$ 、 $R_{2} 、 V_{r e f}$ and $V_{o s}$, and it does not associate with $k_{1} 、 k_{2}$ 、 $k_{3}$, the op-amp's Gain coefficients. Therefore, the impact of temperature drifting on output can be effectively minimized by selecting a low-temperature-drifting $V_{\text {ref }}$ and by controlling $V_{\text {os }}$ of op-amps. For the same reason, a consistent and stable excitation signal $U_{r}\left(u_{r}=u_{o} / k_{2}, k_{2}\right.$ is an ac/dc transforming factor) can be realized by selecting a lock-in amplifier with high noisy-suppression quality.

\section{SYSTEM DESIGN}

\section{A. Design scheme for excitation signal source}

Figure 2 shows that design scheme for the excitation signal source. The square wave input signal $U_{i}$ is first processed by the active filters and the power amplifier to get $U_{r}$, and $U_{r}$ is then processed by lock-in amplifier detector to get linear DC voltage $U_{o} . U_{o}$ is used as a reference voltage for the succeeding A/D conversion. At same time, $U_{o}$ is used as input for the integrating circuit as well to compare with $V_{\text {ref }}$ to get error voltage $U_{e}$, which is further converted by level converter to get $U_{f}$. Finally $U_{f}$ is used as negative feedback input to automatically adjust the amplitude of the square wave of input signal $U_{i}$ to keep $U_{r}$ and $U_{o}$ stable.

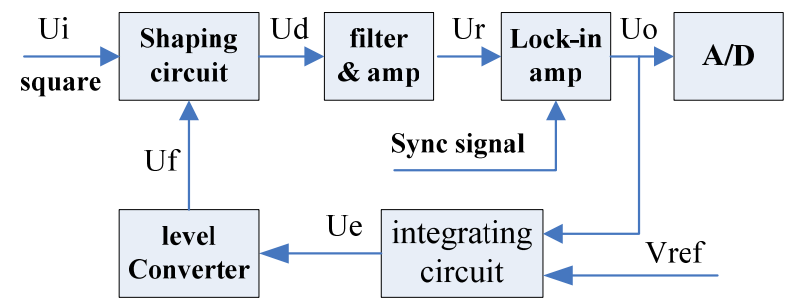

Figure 2 Design scheme of the signal source

\section{B. Module circuit design}

From above design, one can tell that the lock-in amplifier and integrating circuit are key parts of the system; their performance has direct impact on the stability and accuracy of the excitation signal source.

1) Lock-in amplifier

Lock-in amplifier, which structure can be shown in Fig.3, is used to reject differential-mode interference. The core unit of lock-in amplifier is Phase-Sensitivity-Detector (PSD), which demodulates the modulating signals. The output of PSD depends on not only amplitude of input signal, but also the phase difference of input and reference signals.

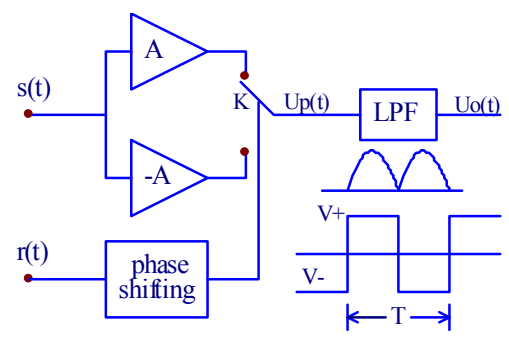

Figure 3. Lock-in amplifier

The following is how the lock-in amplifier works:

Supposing that sinusoidal modulating signal with noise is given as below:

$$
s(t)=u_{0}(t)+n(t)=U_{0} \cos \left(\omega_{0} t+\theta\right)+n(t)
$$

Where $\mathrm{u}_{0}(\mathrm{t})$ is the sinusoidal modulating signal, $\mathrm{n}(\mathrm{t})$ is random noise signal.

Also supposing that the reference input is a square wave with amplitude of $\pm V_{+}$and period of $T$.

Then its Fourier expansion is shown as follow:

$$
r(t)=\frac{4 V_{+}}{\pi} \sum_{n=1}^{\infty} \frac{(-1)}{2 n-1}^{n+1} \cos \left[(2 n-1) \omega_{0} t\right]
$$

The output signal of PSD can be calculated as below:

$$
\begin{aligned}
& u_{p}(t)=s(t) r(t)=\left[u_{0}(t)+n(t)\right] r(t) \\
& =\frac{2 K_{1} U_{0} V_{+}}{\pi} \sum_{n=1}^{\infty} \frac{(-1)}{2 n-1}^{n+1}\left\{\cos \left[(2 n-2) \omega_{0} t-\theta\right]\right. \\
& \left.+\cos \left(2 n \omega_{0} t+\theta\right)+n(t) \cos (2 n-1) \omega_{0} t\right\}
\end{aligned}
$$

Where $\cos \left[(2 \mathrm{n}-2) \omega_{0} \mathrm{t}-\theta\right]$ is the unit of difference frequency, $\cos \left(2 \mathrm{n} \omega_{0} t+\theta\right)$ is the unit of sum frequency, and $\mathrm{n}(\mathrm{t}) \cos \left[(2 \mathrm{n}-1) \omega_{0} t\right]$ is the noise .

After the signal $U_{p}(t)$ passes through low pass filter (LPF), the difference frequency, the sum frequency unit, and the noise all will be filtered out when $\mathrm{n}$ is greater than one. What remains is only the difference frequency $\mathrm{U}_{\mathrm{o}}$ when $\mathrm{n}$ is equal to one.

$$
U_{o}=\frac{2 k_{2} U_{r m} V_{+}}{\pi} \cos \theta
$$

The $U_{r}$ is used as the reference voltage with phase $\theta=0$, so one can get: 


$$
U_{o}=\frac{2 k_{2} U_{r m} V_{+}}{\pi}
$$

From above, one can tell that the accumulated noise in the signal is effectively suppressed. Since $k_{2}$ and $V_{+}$are constants, $U_{o}$ and $U_{r}$ has a linear relation. Figure 4 shows the lock-in amplifier based on the analog switch $74 \mathrm{HC} 4053$ and the RC filters .

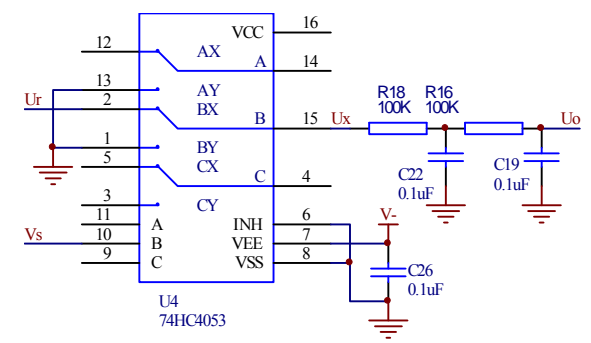

Figure 4 Lock-in amp of the electronic switch

\section{2) Integrating circuit}

Figure 5 shows an integrating circuit. The main interference comes from integrating drift, which is the accumulated electric charge on its capacitors when the input is zero. The integrating drift may result in an output from zero to saturation. The main source of integrating drift is from the input offset voltage $V_{\text {os }}$ of op-amps.

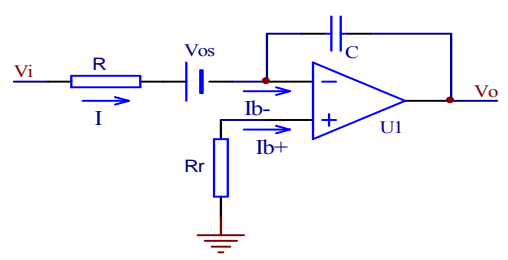

Fig. 5 Integrating circuit

According to KVL, concrete analysis is as follows:

$$
\begin{gathered}
V_{i}=I R+V_{o s}-I_{b+} \times R_{r} \\
V_{o}=-\frac{1}{C} \int\left(I-I_{b-}\right) d t+V_{o s}-I_{b+} R_{r}
\end{gathered}
$$

Combining (12) with (13), one gets the following:

$$
\begin{aligned}
& V_{o}=-\frac{1}{R C} \int V_{i} d t+\frac{1}{R C} \int V_{o s} d t \\
& +\frac{1}{R C} \int I_{b-} R_{r} d t+V_{o s}-I_{b+} R_{r}
\end{aligned}
$$

Where $I_{b+} 、 I_{b-}$ are the bias currents for non-inverting input and inverting input correspondingly. $I_{o s}=I_{b-}-I_{b+}$, the input offset current of op-amps, and $-\frac{1}{R C} \int V_{i} d t$ is the ideal linear output, the rest is the integrating drift by integrator.
It can be seen that the output error are mainly caused by $V_{o s}$ and $I_{o s}$, since $V_{o s}$ and $I_{o s}$ are convertible with each other. Usually only $V_{o s}$ is for analyzing.

From above analysis and equation (6), Output $U_{o}$ is decided by $R_{1} 、 R_{2} 、 V_{\text {ref }}$, and $V_{o s}$. For $R_{1}$ and $R_{2}$, a high quality metal film resistor can completely meet the design requirement, therefore $V_{\text {ref }}$ and $u_{o s}$ are critical factors for drift suppression.

In the design, we selected OP400 of AD Cooperation mainly for its low-offset. For a stable reference voltage $u_{\text {ref }}$, LM336-2.5 of NS Corporation was selected due to its low temperature drifting. Figure 6 is the actual design of the integration circuit 。

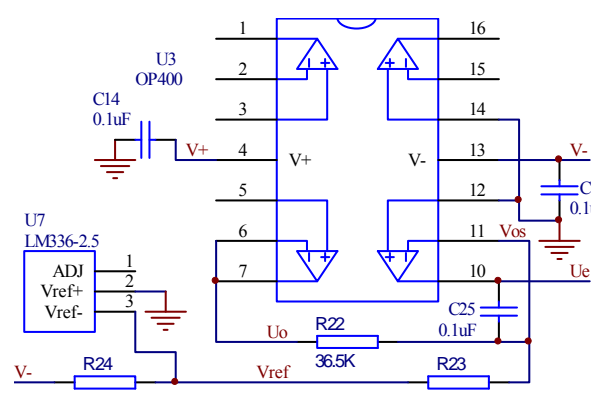

Fig. 6 Integrating circuit application

\section{3) Wave Shaping circuit}

As shown in Figure 7, wave shaping is accomplished by inverters. With 74HC14, the amplitude of output square wave is automatically adjusted. Integrating error signal $u_{e}$ is converted into a feedback signal $u_{f}$ to control Vss so that the voltage source for the circuit is under control.

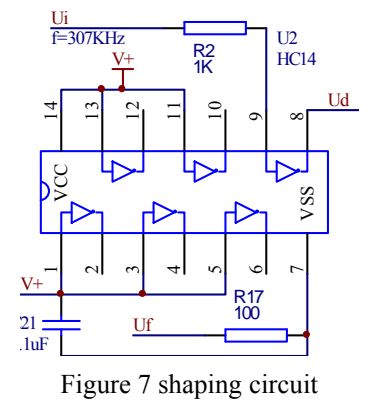

The amplitude of output square wave is:

$$
U_{A}=k\left(V_{C C}-V_{S S}\right)
$$

Where $\mathrm{K}$ is the proportion factor, $V_{c c}$ is the voltage source. Supposing that $U_{i}$ would go up when temperature goes up, but $U_{d}$ can be kept nearly unchanged by the negative feedback process. Here is how it works: 
$U_{i} \uparrow \rightarrow U_{d} \uparrow \rightarrow U_{o} \uparrow \rightarrow U_{e} \downarrow \rightarrow U_{f} \uparrow \rightarrow\left(V_{C C}-V_{S S}\right) \downarrow \rightarrow U_{d} \downarrow$

\section{THE RESULTS AND ITS ANALYSIS}

We designed an excitation signal source and used it in the application of thickness measurement for PCB copper foil. Its frequency is $307 \mathrm{KHz}$. The tests on $U_{r} 、 U_{o}$ and $V_{r e f}$ were performed with temperature range from $0^{\circ} \mathrm{C}$ to $+70^{\circ} \mathrm{C}$.

Figure 8 is the actual $U_{r}$ wave when the temperature is $25^{\circ} \mathrm{C} . U_{r}=1.05 \mathrm{~V}, U_{o}=0.4767 \mathrm{~V}$, and $V_{r e f}=-2.485 \mathrm{~V}$ 。

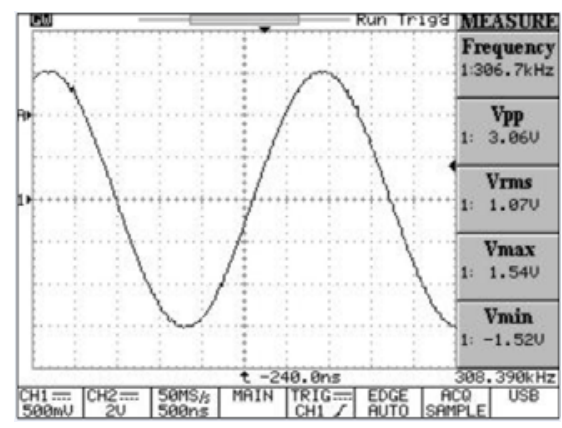

Figure. 8 Waveform of Ur

Under the condition of $\mathrm{V}+=5 \mathrm{~V} 、 \mathrm{~V}-=-5 \mathrm{~V}$, the measurements on $U_{r} 、 U_{o}$ and $V_{\text {ref }}$ were performed at each of following temperature points: $0^{\circ} \mathrm{C}, 25^{\circ} \mathrm{C}, 50^{\circ} \mathrm{C}$, and $70^{\circ} \mathrm{C}$, and the temperature was kept at each specific point for 30 minutes. The table one shows the results:

TABLE. 1 TEST DATA

\begin{tabular}{|c|c|c|c|}
\hline $\mathrm{T}\left({ }^{\circ} \mathrm{C}\right)$ & $\mathrm{Ur}(\mathrm{V})$ & Uo $(\mathrm{V})$ & $\operatorname{Vref}(\mathrm{V})$ \\
\hline 0 & 1.05 & 0.4766 & -2.486 \\
\hline 25 & 1.05 & 0.4767 & -2.485 \\
\hline 50 & 1.05 & 0.4768 & -2.483 \\
\hline 70 & 1.05 & 0.4770 & -2.482 \\
\hline
\end{tabular}

From above table, it shows that $U_{r}$ does not change when the temperature changes from $0^{\circ} \mathrm{C}$ to $+70^{\circ} \mathrm{C}$; since the output
DC voltage $\Delta U_{o}=0.4 m V$, from equation (6), $\Delta U_{o}$ is caused by $\Delta V_{\text {ref }}=4 m V$, this would ensure a stable excitation signal $U_{r}$ and a stable and accurate $U_{o}$ as a base voltage for $\mathrm{A} / \mathrm{D}$ conversion 。

\section{CONCLUSION}

In this paper, an excitation signal source with effective temperature-drifting suppression is designed for eddy current testing. The temperature drifting problem in op-amps is studied as well. The mathematical model is established for a improvement of the negative feedback system. Based on the analysis of the mathematical model, the system and its circuits are designed with lock-in amplifier and integrating circuit as the core parts of the system for temperature-drifting suppression. The test results indicate that this approach and its design is very effective in suppressing temperature drifting. The excitation signal and output reference voltages are very stable. This ensures a consistent and accurate thickness measurement results for PCB copper foil.

We believe that the study and design of an improved excitation signal source in this paper can be applied in other applications as well.

\section{REFERENCES}

[1] McDonough R N \& Whalen A D. Detection of Signals in Noise. Second Edition. New York: AT\&T Bell Laboratories and Academic Press, 1995

[2] Mohanty N. Random Signals Estimation and Identification. New York: Van Nostrand Reinhold Company Inc. , 1986

[3] Schulz R B, et al. Shielding Theory and Practice. IEEE Transaction on Electromagnetic Compatibility. 1988, 30(3):187-201

[4] Jiri Jan. Digital Signal Filtering, Analysis and Restoration. London: IEEE, 2000

[5] Gersho A. Adaptive Filtering with Binary Reinforcement. IEEE Transactions on Information Theory, 1984, IT-30(2): 191-198

[6] Ott H W. Electromagnetic Compatibility Engineering. Wiley, 2009

[7] Davies E R. Electronics Noise and Signal Recovery. London: Academic Press Limited, 1993

[8] Witteman W J. Detection and Signal Processing: Technical Realization. Berlin: Springer, 2006

[9] Vasilescu G. Electronic Noise and Interfering Signals: Principles and Applications. Berlin Heidelberg: Springer-Verlag, 2005 